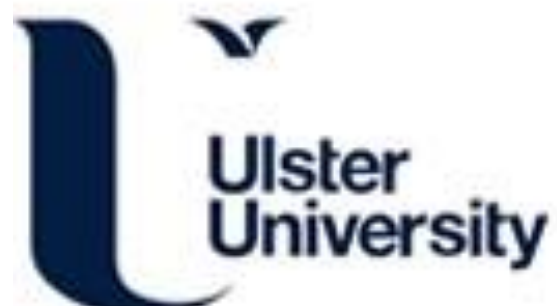

Voice pathology detection using interlaced derivative pattern on glottal source
excitation

Muhammad, G., Alsulaiman, M., Ali, Z., Mesallam, T. A., Farahat, M., Malki, K. H., Al-nasheri, A., \& Bencherif, M. A. (2017). Voice pathology detection using interlaced derivative pattern on glottal source excitation.

Biomedical Signal Processing and Control, 31, 156-164. https://doi.org/10.1016/j.bspc.2016.08.002

Link to publication record in Ulster University Research Portal

Published in:

Biomedical Signal Processing and Control

Publication Status:

Published (in print/issue): 31/01/2017

DOI:

10.1016/j.bspc.2016.08.002

Document Version

Author Accepted version

\section{General rights}

Copyright for the publications made accessible via Ulster University's Research Portal is retained by the author(s) and / or other copyright owners and it is a condition of accessing these publications that users recognise and abide by the legal requirements associated with these rights.

\section{Take down policy}

The Research Portal is Ulster University's institutional repository that provides access to Ulster's research outputs. Every effort has been made to ensure that content in the Research Portal does not infringe any person's rights, or applicable UK laws. If you discover content in the Research Portal that you believe breaches copyright or violates any law, please contact pure-support@ulster.ac.uk. 


\title{
Voice Pathology Detection Using Interlaced Derivative Pattern on Glottal Source Excitation
}

Ghulam Muhammad $^{1, *}$, Mansour Alsulaiman ${ }^{1}$, Zulfiqar Ali ${ }^{1,2}$, Tamer A. Mesallam ${ }^{3,4,5}$, Mohamed Farahat ${ }^{3,4}$, Khalid H. Malki ${ }^{3,4}$, Ahmed Al-nasheri ${ }^{1}$, Mohamed A. Bencherif ${ }^{1}$

${ }^{1}$ Digital Speech Processing Group, Department of Computer Engineering, College of Computer and Information Sciences, King Saud University, Riyadh 11543, Saudi Arabia.

${ }^{2}$ Centre for Intelligent Signal and Imaging Research (CISIR), Department of Electrical and Electronic Engineering, Universiti Teknology PETRONAS, Tronoh 31750, Perak, Malaysia.

${ }^{3}$ ENT Department, College of Medicine, King Saud University, Riyadh, Saudi Arabia.

${ }^{4}$ Research Chair of Voice, Swallowing, and Communication Disorders, King Saud University, Riyadh, Saudi Arabia.

${ }^{5}$ ENT Department, College of Medicine, Menoufiya University, Shebin Alkoum, Egypt.

Keywords: Interlaced derivative pattern (IDP), AVPD, SVD, MEEI, Voice pathology detection, Glottal source excitation

\begin{abstract}
:
In this paper, we propose a voice pathology detection and classification method using an interlaced derivative pattern (IDP), which involves an $n$-th order directional derivative, on a spectro-temporal description of a glottal source excitation signal. It is shown previously that directional information is useful to detect pathologies due to its encoding ability along time, frequency, and timefrequency axes. The IDP, being an $n$-th order derivative, is capable of describing more information than a first order derivative pattern by combining
\end{abstract}


all the directional information into one. In the IDP, first-order derivatives are calculated in four directions, and these derivatives are thresholded with the center value of each directional channel to produce the final IDP. A support vector machine is used as a classification technique. Experiments are conducted using three different databases, which are the Massachusetts Eye and Ear Infirmary database, Saarbrucken Voice Database, and Arabic Voice Pathology Database. Experimental results show that the IDP based features give higher accuracy than that using other related features in all the three databases. The accuracies using cross-databases are also high using the IDP features.

\section{INTRODUCTION}

Automatic detection of vocal fold pathologies is an interest to the researchers of speech or voice community, as well as the respective medical community. This is due to its non-invasive nature, free from subjective biasness, and relatively low cost. There have been a lot of researches to detect voice pathology by analyzing voice. The main emphasis was to develop a feature or a feature vector that can effectively distinguish between normal and pathological voices. The features can broadly be divided into two groups, one imported from speech or speaker recognition applications, and the other from voice quality measurements. The features that came from speech or speaker recognition applications include Mel-frequency cepstral coefficients (MFCC), linear prediction cepstral coefficients (LPCC), and relative spectra per- ceptual linear prediction (RASTA-PLP) [1-3]. On the other hand, the features coming from voice quality measurements are, among others, shimmer, jitter, harmonic-to-noise ratio, and cepstral peak prominence [3]. Recently, features from audio and image process- ing applications are also integrated in voice pathology detection. These features include MPEG-7 audio features [4], fractal analysis [5], modulation spectrum [6], formants [35]. The use of nonlinear features have studied too in the field of voice pathology detection [29-32].

Though there exists a lot of related works, most of them use the Massachusetts 
Eye and Ear Infirmary (MEEI) database [7], where normal and pathological samples are recorded in two different environments. Therefore, for a classification technique, it is not clear whether the classifier is classifying pathologies or environments. There are some works that use other databases; however, many of them are using only one database at a time. The question arises: are the existing voice pathology detection techniques database dependent? For example, Markaki and Stylianou showed that normalized modulation spectral features achieve more than $92 \%$ detection rate in the MEEI database, but it drops below $80 \%$ in a cross-database experiment [8]. The same features set without normalization have a detection rate of $94.1 \%$ in the MEEI database, and $62.3 \%$ in cross-database. The normalization increases the accuracy in the cross-database scenario at the expense of a decrease in a single database experiment. There- fore, there is a need to develop such a features set that achieve a high accuracy in both single database and cross-database experiments. These features should also visually justify the detection.

In this paper, a set of features are proposed to detect voice pathology from a glottal source excitation signal. A voice signal is a convolution between a source signal (originated from the lung) and the vocal tract filter. In a voiced signal, the vocal folds open and close in a periodic manner that produces pitch of the signal. The vocal tract is simply shaping the signal to produce a desired phone. In this study, we refer voice pathology to an abnormality in voice caused by pathology in the vocal fold(s). Vocal folds contribute to the glottal source excitation, and therefore, any abnormality in the glottal source excitation is an indication of voice pathology. There are some researches of voice pathology detection using the glottal source excitation [9-12]. For example, glottal formant frequency and bandwidth, spectral balances, and center of gravity are extracted from the glottal source signal to detect voice pathology in [10]. Features from dynamics of average glottal source and mucosal wave spectrum are used to detect voice pathology in [11]. In [9], a power spectral density envelop of the glottal source is utilized in a form of a specific harmonic-to-harmonic relationship. Back in 1975, Koike and Markel first showed the use of inverse filtering (residue output) in voice pathology detection in an experiment involving only 10 normal 
persons and 10 patients having voice pathology [12]. Residue signal is also used in [13] for voice pathology detection. There are several techniques to estimate the glottal source excitation from the voice signal. A good review of this topic can be found in [14].

In this paper, the first-order derivative of the glottal source excitation is utilized to extract features. It is well-known that a first-order derivative (without smoothing) generally decreases the signal-to-noise ratio. Any weak disturbances caused by irregular vibrations of the vocal folds (due to the location and the shape of the pathology, elasticity of the tissues, etc.) are emphasized in the first- order derivative signal. This derivative signal is further processed to have a spectrum. The spectrum is divided into several bands, whose center frequencies are 'Mel' spaced. An interlaced derivative pattern (IDP) $[15,16]$ is extracted from the spectro-temporal representation of the first-order derivative of the glottal source. The IDP is a texture descriptor, and successfully applied to applications including gender recognition from faces [15] and automatic speech recognition [16]. A support vector machine (SVM) is applied for classification. Three different databases are used to evaluate the proposed method for voice pathology detection and classification. The major contributions of this paper are (i) to develop a method using the IDP to detect and classify voice pathologies, (2) to use first-order derivative in the glottal source excitation to lower the signal-to-noise ratio to enhance the weak noises arisen from the closing and opening of the pathological vocal folds, (3) to validate the performance of the proposed method using multiple databases, and using cross-database, and (4) to investigate the use of the voice signal and the glottal source excitation by an inverse filtering in the proposed method.

The rest of the paper is organized as follows. Section 2 describes the proposed method; Section 3 presents the experiments; Section 4 gives the discussion, and Section 5 draws some conclusions. 


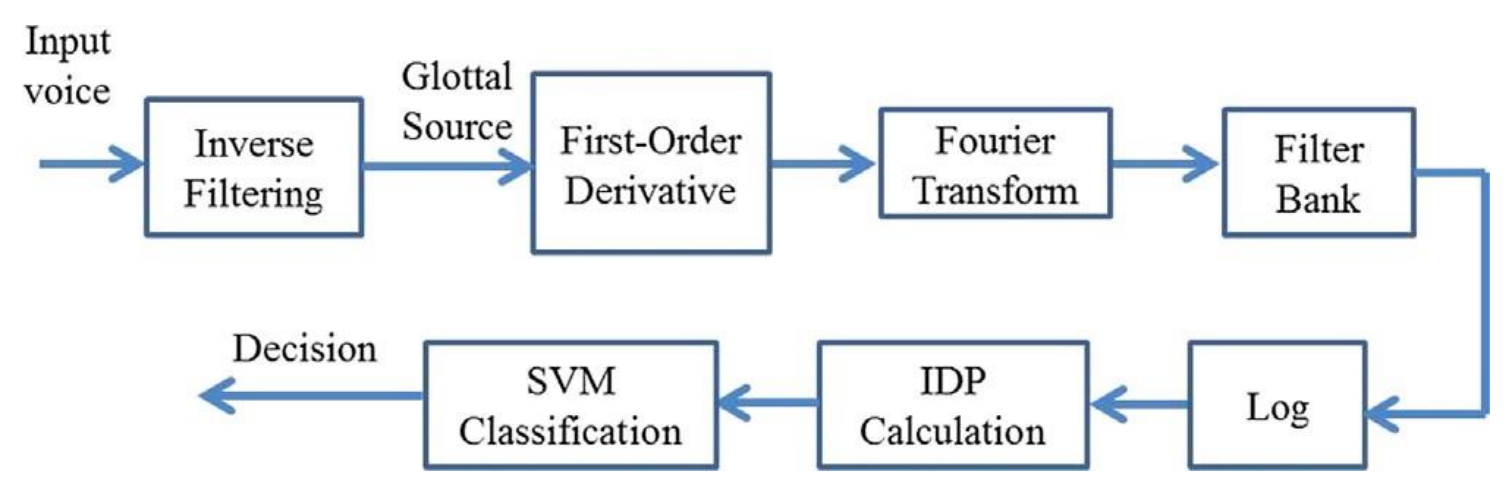

Figure 1: Block diagram of the proposed IDP based voice pathology detection and classification method.

\section{PROPOSED METHOD}

Fig. 1 shows a block diagram of the proposed IDP based voice pathology detection and classification method. First, a glottal source excitation signal is estimated using an interactive adaptive inverse filtering (IAIF) technique from an input voice signal. First-order derivative of the glottal signal is calculated for further processing. The Fourier transform is applied to the frames of the first-order derivative signal to get a spectrum. 24 band-pass filters (BPFs), whose center frequencies are spaced on a Mel scale, are utilized to get the contributions of 24 different frequency bands. The filter outputs are then log-compressed. The IDP encodes an $n$-th order directional derivative pattern of the spectro-temporal description (whose horizontal axis represents the frame number, and vertical axis represents the filter number). The IDP features are fed to an SVM classifier.

\subsection{Glottal source excitation}

There are several techniques to estimate the glottal source excitation. In this study, we used the IAIF algorithm [17], which is publicly available at http://users.aalto.fi/ traitio/research.html. In the IAIF algorithm, high-pass filtering is applied to remove low 
frequency fluctuations. Using the linear predictive coding, the effect of the vocal tract filter is estimated, and is canceled out through inverse filtering. The effect of the lip radiation is canceled through integration. This process is done iteratively to get a good estimation of the glottal source [17]. By inverse filtering, the effect of vocal tract filtering is eliminated. We do not need spectral information of vocal tract filtering, because we are concerned about glottal signal (which is affected by vocal fold pathologies), not speech characteristics. The spectral characteristics that we are interested in are produced by irregular vibrations of the vocal folds, not the spectral characteristics produced by the vocal tract shaping.

Once the glottal source waveform is estimated, a first-order derivative operation is applied to the waveform to get the derivative wave. This derivative wave has a lower signal-to-noise ratio, which is useful to find the weak noise contribution that arises from vocal fold pathologies. For a good phonation, energy is mostly concentrated in lower frequencies due to low glottal formant frequencies; however, because of irregular vibration of the vocal folds in case of pathology, energy is significant also in higher frequencies. Fig. 2 shows examples of the spectrums of the glottal source signal and its first-order derivative signal of a pathological sample. From the figure, we can see that in the higher frequencies there is more energy in the first-order derivative signal spectrum than in the original glottal source signal (see the zoomed-in frequency range [2000-4000] Hz in (e) and (f) of Fig. 2). This justifies the use of firstorder derivative signal for further processing. The signal is framed, where the frame size is 30 milliseconds, and Hamming windowed. The frames are overlapped by $50 \%$. The Fourier transform is applied to each frame to get a spectrogram from the firstorder derivative signal. 24-BPFs are applied to the spectrogram. From experiments, we found that outputs of filters 17-24 are not discriminative to this task; therefore, we retained the outputs of the first 16 filters. 


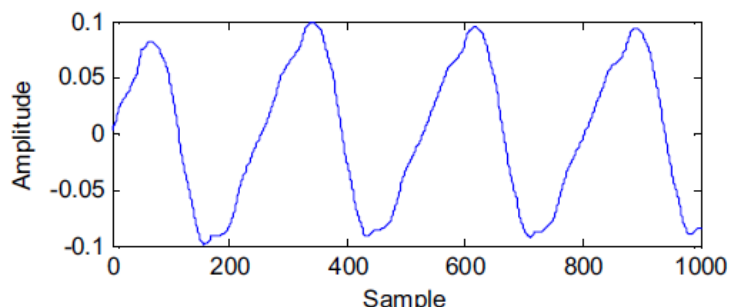

(a) Glottal source excitation signal

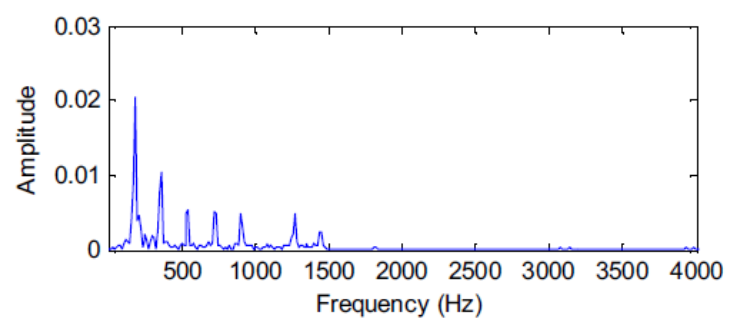

(c) Spectrum of glottal excitation signal

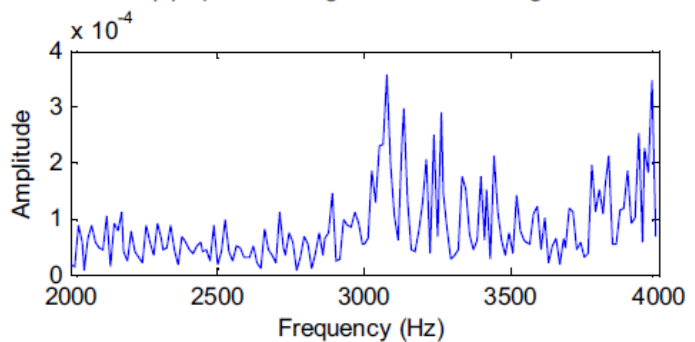

(e) Zoomed part of (c) in the range [2000 - 4000] Hz

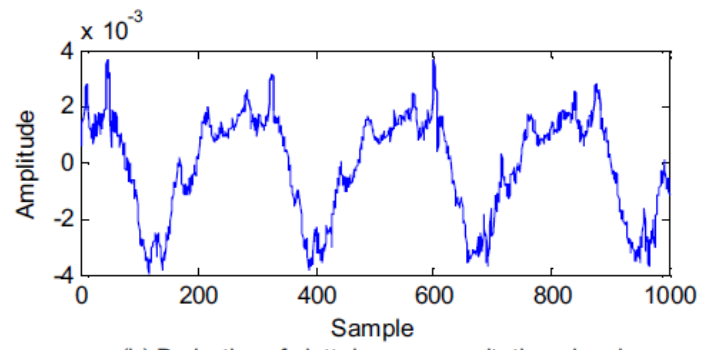

(b) Derivative of glottal source excitation signal

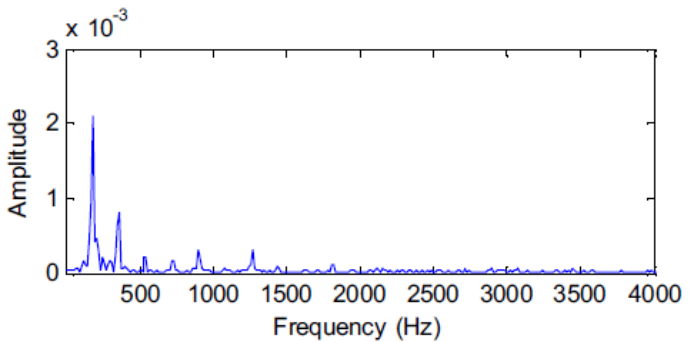

(d) Spectrum of derivative of glottal source excitation signal

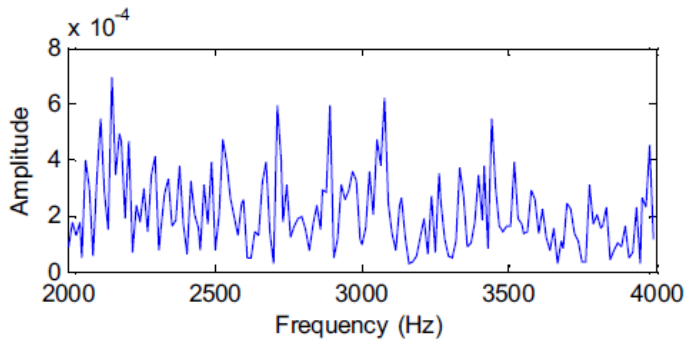

(f) Zoomed part of (d) in the range [2000 - 4000] Hz

Figure 2: Difference of spectrums of glottal source excitation signal and its derivative signal. By comparing (e) and (f) we see that there is higher energy at [2000-4000] Hz in the spectrum of the derivative of the glottal source excitation signal than that of the glottal source excitation signal.

\subsection{IDP}

We have shown before in [18] that directional derivative, which we named as multidirectional regression (MDR), on a spectro- temporal pattern is useful in voice pathology detection. Here, we extend it to a second-order derivative that can encode different directional patterns in a single pattern.

The IDP is a powerful local texture pattern descriptor $[15,16]$. It is less affected by noise and less redundant than other local texture pattern operators, such as the local binary pattern (LBP) [19]. The $n$-th order IDP operator can be expressed as follows: 
$I D P^{n}\left(P_{0}\right)=\left\{\begin{array}{l}f\left(M_{135}{ }^{n-1}\left(p_{0}\right), M_{135}{ }^{n-1}\left(p_{1}\right)\right), f\left(M_{90}{ }^{n-1}\left(p_{0}\right), M_{90}{ }^{n-1}\left(p_{2}\right)\right), \\ f\left(M_{45}{ }^{n-1}\left(p_{0}\right), M_{45}{ }^{n-1}\left(p_{3}\right)\right), f\left(M_{0}^{n-1}\left(p_{0}\right), M_{0}{ }^{n-1}\left(p_{4}\right)\right), \\ f\left(M_{135}{ }^{n-1}\left(p_{0}\right), M_{135}{ }^{n-1}\left(p_{5}\right)\right), f\left(M_{90}{ }^{n-1}\left(p_{0}\right), M_{90}{ }^{n-1}\left(p_{6}\right)\right), \\ f\left(M_{45}{ }^{n-1}\left(p_{0}\right), M_{45}{ }^{n-1}\left(p_{7}\right)\right), f\left(M_{0}{ }^{n-1}\left(p_{0}\right), M_{0}{ }^{n-1}\left(p_{8}\right)\right)\end{array}\right\}$

where the function $f$ is defined as

$f(x, y)=\left\{\begin{array}{l}1, \text { if }(x-y) \geq 0 \\ 0, \text { if }(x-y)<0\end{array}\right.$

In the proposed method, an IDP is produced for the spectro-temporal (spectrogram) description of the first-order derivative of the glottal source. The spectro-temporal description can be considered as an image. The IDP is a four-channel derivative image, representing four directional $n$-th order derivative in $0^{\circ}, 45^{\circ}, 90^{\circ}$, and $135^{\circ}$, respectively. These directions capture temporal, rising temporal-frequency, frequency, and falling temporal-frequency, respectively, information of the spectrogram. This information is further enhanced by taking one more order derivative. The order of derivatives is derived from the order of the IDP operator; i.e., for an n-th order IDP operator, the IDP images with four (n-1)-th order derivative channels are produced. These derivative channels present more detailed description of the spectrogram in four directions. A $3 \times 3$ neighborhood is selected around each point in the spectrogram. For each neighbor, the direction between the center and the neighbor is computed, and the IDP channel with the same direction is selected [15], [16]. Fig. 3 illustrates the 2nd-order IDP operator.

The neighbor is thresholded with the center value in the selected IDP channel, and the result is encoded as a binary number. This thresholding actually encodes the binary result of the first-order derivative among local neighbors and produces an extra order to the IDP operator. Therefore, in each direction, only the derivatives for the center point and its neighbor point in that particular direction will be calculated. The IDP encodes the relationships in the particular directions. In this way, the IDP keeps only the more important information and makes the process much faster. It produces an 8-bit representation of each value of the Melspectrogram. The spectro-temporal directional derivative pattern [20], multi directional 


\begin{tabular}{|c|c|c|c|c|}
\hline 8 & 3 & 7 & 4 & 1 \\
\hline 9 & 7 & 8 & 2 & 10 \\
\hline 3 & 3 & 5 & 4 & 7 \\
\hline 7 & 9 & 10 & 8 & 8 \\
\hline 6 & 5 & 4 & 9 & 2 \\
\hline \multicolumn{5}{c|}{$\mathrm{P}_{0}$} \\
\hline
\end{tabular}

(a)

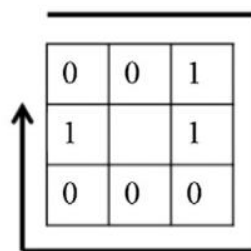

(c)

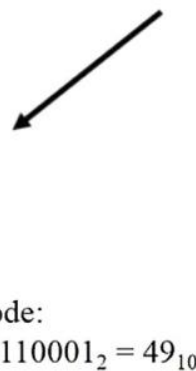

$00110001_{2}=49_{10}$

\begin{tabular}{|c|c|c|c|c|c|}
\hline-1 & 6 & -8 & 0 & 4 & 1 \\
\hline-2 & 1 & -3 & -5 & 3 & -6 \\
\hline-1 & 2 & 0 & 4 & 6 & 1 \\
\hline \multicolumn{3}{|c|}{ Channel $0^{\circ}$} & \multicolumn{3}{|c|}{ Channel $45^{\circ}$} \\
\hline 4 & 1 & -2 & -1 & 5 & -5 \\
\hline-4 & -3 & 2 & -6 & -2 & -4 \\
\hline 6 & 5 & 4 & 6 & 7 & 3 \\
\hline \multicolumn{3}{|c|}{ Channe } & \multicolumn{3}{|c|}{ Channel $135^{\circ}$} \\
\hline
\end{tabular}

(b)

Fig. 3. Illustration of the IDP calculation: (a) a spectro-temporal pattern, where horizontal axis and vertical axis represent frame and filter, respectively, (b) first-order derivative along four directions, and (c) final IDP value for the central element $\mathrm{P}_{0}$. 
derivative pattern [21], and LBP [5] were applied to speech processing applications before; however, they provide first-order derivative information and are incapable of describing more detailed information, while the IDP gives $n$-th order derivative that can encode more information in a compact form. This is because the amplitude of the $\mathrm{n}$-the derivative of a peak-shaped signal is inversely proportional to the $n$-th power of the width of the signal. The higher the value of $n$ is, the more discrimination between a narrow spectral peak and a broad spectral peak is. To restrict an increase in computation, we use 2nd-order IDP. Fig. 4 shows examples of the IDP and first-order derivative of the glottal source excitation for (a) a normal sample, (b) a pathological sample of vocal fold cyst, (c) a pathological sample of vocal fold polyp, and (d) a pathological sample of vocal fold paralysis. From the figure, it is obvious that there is more energy (yellow color dominant) in the high number of filters (filters 8-16) in the pathological sample and less energy (red color dominant) in the normal sample. Also, the distribution of energy in different filters for various pathology types is different. In case of polyp, high filters have less energy (red color), and filters 3-8 have high energy (green color). In case of cyst, filters 5-14 have medium energy (yellow color), and in case of paralysis, high energy is present in filters 10-14. So, the difference is visually distinguishable, which may help a doctor to have a primary guess of the pathology type. After this stage, we have 16 features per frame to feed into the SVM classifier. 

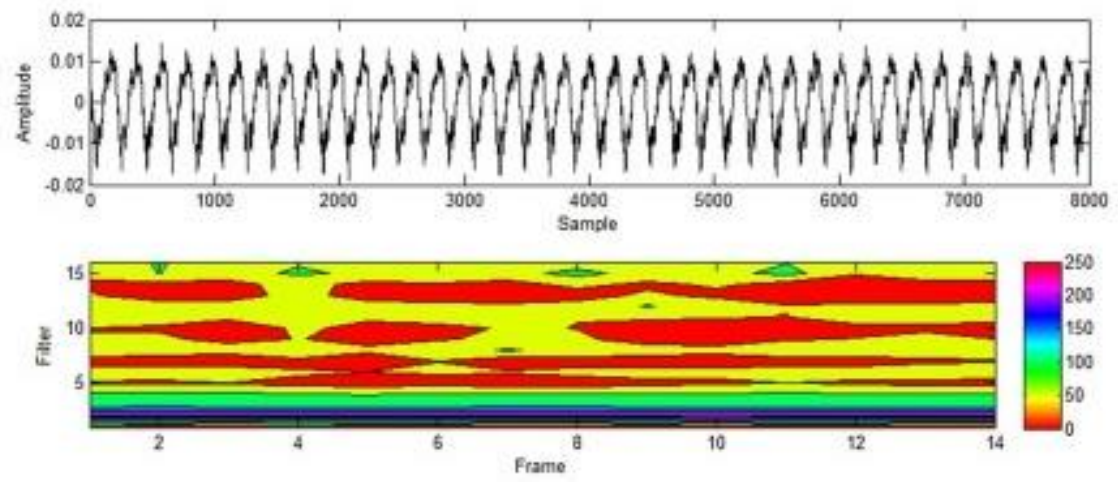

(a) For a normal sample. Top row: $1^{\text {st }}$-order derivative of a glottal source; bottom row: the IDP.
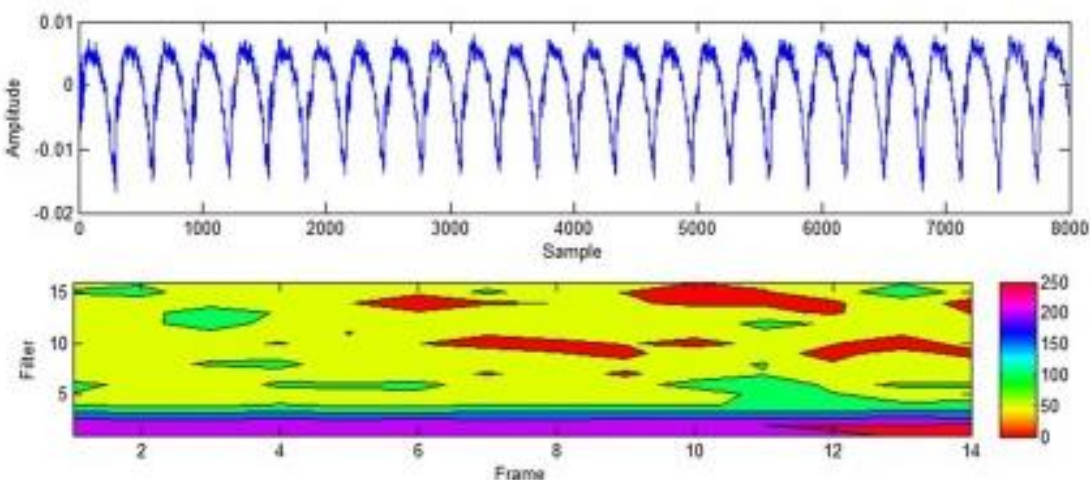

(b) For a pathological (vocal fold cyst) sample. Top row: $1^{\text {st }}$-order derivative of a glottal source; bottom row: the IDP. 

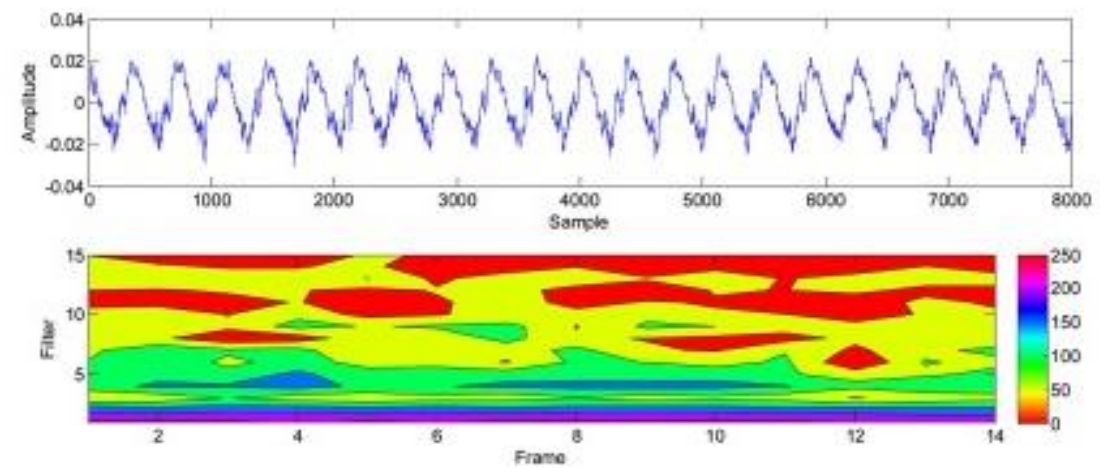

(c) For a pathological (vocal fold polyp) sample. Top row: $1^{\text {st }}$-order derivative of a glottal source; bottom row: the IDP,
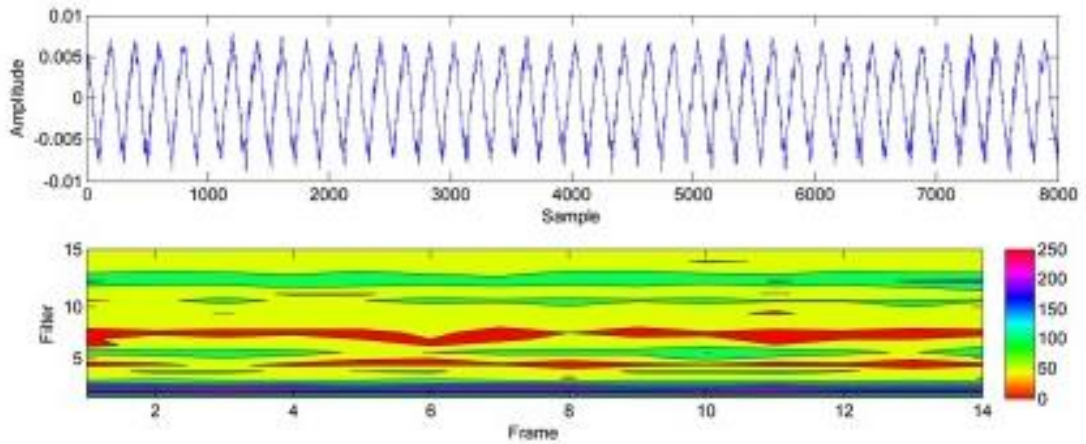

(d) For a pathological (vocal fold paralysis) sample. Top row: $1^{\text {st }}$-order derivative of a glottal source; bottom row: the IDP.

Figure 4: Examples of the IDP for a normal and different types of pathological samples.

\section{EXPERIMENT}

\subsection{Database}

Three databases were used for the experiments to evaluate the robustness of the proposed method. The databases are the MEEI database, Saarbruecken Voice Database (SVD) [22], and Arabic Voice Pathology Database (AVPD). The speakers in MEEI database samples are native English, those in SVD are native German, while those in AVPD are native Arabs. Only sustained vowel/a/samples were used. Three pathologies, which are vocal folds cysts, unilateral vocal fold paralysis, and 
vocal fold polyp, are chosen because these three are common in all the three databases. The number of samples and the gender distribution are given in Table 1. All the files, where necessary, were downsampled to have a uniform sampling frequency of $25 \mathrm{KHz}$.

We chose the MEEI database, because it is the most commonly used database in voice pathology detection research despite its several limitations. The SVD is a freely downloadable database [22]. It has been recorded by the Institute of Phonetics of Saarland University in Germany. This database contains sustained vowels /a/, /i/ and /u/ with different intonations, normal, low, high and low-high-low, spoken by a large number of normal persons and patients having voice pathology. Very few studies of voice pathology detection have been done on this database [23].

Table 1: Normal and pathological samples (No. of Male speakers + No. of Female Speakers) from three different databases.

\begin{tabular}{|c|c|c|c|c|c|}
\hline \multirow{2}{*}{ Database } & \multirow{2}{*}{ Normal } & \multicolumn{4}{|c|}{ Pathological } \\
\cline { 3 - 6 } & & Cysts & Paralysis & Polyp & Total \\
\hline AVPD & $118(93+25)$ & $13(7+6)$ & $32(16+16)$ & $30(14+16)$ & 75 \\
\hline MEEI & $53(21+32)$ & $10(6+4)$ & $70(38+32)$ & $15(8+7)$ & 95 \\
\hline SVD & $\begin{array}{c}262(137+ \\
125)\end{array}$ & $6(5+1)$ & $\begin{array}{c}194(121+ \\
73)\end{array}$ & $44(19+25)$ & 244 \\
\hline
\end{tabular}

\subsubsection{Arabic Voice Pathology Database (AVPD)}

The MEEI database has a limitation in the sense that the pathological samples and the normal samples were recorded in two different environments. Therefore, in the experiment with the MEEI database, it is not clear whether the system detects pathology or environment. With the support of National Plans for Science and Technology (NPST), Saudi Arabia, we started to develop AVPD that contains samples of sustained vowels, Arabic digits, some common words, and paragraphs. The selected text covers all Arabic phonemes. All speakers record three utterances of each vowel $/ \mathrm{a} /, / \mathrm{u} /$ and $/ \mathrm{i} /$, while isolated words and continuous speech are recorded once to avoid making a burden on patients. All the speakers were clinically assessed. In case of pathology, the 
perceptual severity of voice disorders was rated on a scale of $1-3$, where 3 represents the most severe case. This severity rating was associated to each sample naming upon consensus of a panel of three expert medical doctors. A total of five types of vocal folds pathologies were considered including sulcus, nodules, polyp, cyst, and paralysis The samples were collected in different sessions at Communication and Swallowing Disorders Unit, King AbdulAziz University Hospital, King Saud University, Riyadh, Saudi Arabia, by experienced phoniatricians in a sound-treated room using a standardized recording protocol. The sampling frequency is $50 \mathrm{KHz}$ (which we downsampled to recorded by using Kay Pentax computerized speech lab (CSL Model 4300). The AVPD will be made publicly available by the end of 2015 .

\subsection{Classification}

For detection and classification, the SVM with radial basis function (RBF) as a kernel was used [24]. There are several advantages of the SVM such as (1) it can provide a good out-of-sample generalization, if the parameters are properly chosen, (2) it can produce a unique solution, unlike neural networks, because the optimality problem is convex, and (3) with the help of kernels, it can achieve the flexibility of the form of the threshold separating the classes. Moreover, the SVM has been extensively utilized in voice pathology detection research [4], [6], [8], [26], [28], [33]. As the principle of SVM is heavily discussed in the literature, we do not provide the details of it in this paper. The RBF was used because it has less restriction than other kernels and is more general than the linear kernels. For a single database experiment, five-fold cross validation approach was utilized, where there was no overlap between training and testing samples in a fold. In the five-fold crossvalidation, the normal samples and the pathological samples are randomly divided into five equal groups each. In each iteration, four groups each from the normal and the pathological samples are used for training, while the remaining samples are used for testing. Therefore, at the end of five iterations, all the five groups are tested. The optimization parameter (C) and the kernel parameter $(\gamma)$ were selected by using an 
extensive grid search on training samples. The average values of $\mathrm{C}$ and $\gamma$ in our experiments were 2.5 and 0.192, respectively. The overall accuracy (ACC), sensitivity ( $\mathrm{SN})$, specificity (SP), and area under the receiver operating characteristic curve (AUC) were obtained by averaging over five folds. For cross-database experiments, there was no fold, because training and testing samples were from different databases. In case of classification experiments, one-versus-rest approach was utilized. As we are using frame level features, the decision on a file is taken based on the majority frames. For example, if the majority of frames in a voice signal are classified as normal, the voice signal is classified as normal.

\subsection{Results}

\subsubsection{Pathology detection}

The results on the pathology detection are given in Table 2. The proposed method is compared with 12 MFCC features plus their velocity coefficients [1], [25], and 22 MDVP parameters [26]. From the table, we see that the proposed IDP based method outperforms the other two in all the three databases. In case of SVD and AVPD, the improvement of the proposed method is significant. The proposed method achieves average accuracies of $99.2 \%$, 93.2\%, and $91.5 \%$ by using MEEI database, SVD, and AVPD, respectively (confidence interval 95\%, $\alpha=0.05$ ). Table 3 shows the detection accuracies in cross-database experiments. There were nine experiments for each of the methods. Six experiments were with one database training and another database testing, while the remaining three were with two databases training and the third database testing. From Table 3, we find that training with MEEI database does not provide a good result, because the system is not well trained for normal and pathology for their different recording environments. The proposed method achieves a good detection accuracy when the system was trained with SVD or AVPD. When the system was trained with two databases, the accuracy of the proposed method goes past $87 \%$ in all the three cases; however, those for the other two methods are still below $77 \%$. 
Table 2. Results of pathology detection (95\% confidence interval). (sd, standard deviation).

\begin{tabular}{|c|c|c|c|c|c|}
\hline Database & Methods & $\%$ ACC \pm sd & $\% S N$ & $\%$ SP & AUC \\
\hline MEEI & Proposed & $99.4 \pm 0.02$ & 99.4 & 98.9 & 0.99 \\
\hline & MFCC 24 & $97.6 \pm 0.19$ & 96.2 & 92.1 & 0.95 \\
\hline & MDVP 22 & $84.3 \pm 1.23$ & 83.2 & 65.2 & 0.85 \\
\hline \multicolumn{7}{|l}{} \\
\hline SVD & Proposed & $93.2 \pm 0.01$ & 94.3 & 92.3 & 0.94 \\
\hline \multicolumn{7}{|l|}{ MFCC 24 } & $75.4 \pm 0.12$ & 74.5 & 76.1 & 0.76 \\
\hline & MDVP 22 & $77.5 \pm 0.78$ & 72.1 & 82.4 & 0.78 \\
\hline AVPD & Proposed & $91.5 \pm 0.09$ & 92.2 & 91.1 & 0.92 \\
\hline & MFCC 24 & $80.6 \pm 1.43$ & 79.1 & 81.2 & 0.81 \\
\hline & MDVP 22 & $79.6 \pm 2.21$ & 72.6 & 84.2 & 0.80 \\
\hline
\end{tabular}

Table 3. Pathology detection accuracies (\%) using cross-database (95\% confidence interval).

\begin{tabular}{|c|c|c|c|c|c|c|c|c|c|c|}
\hline & & \multicolumn{9}{|l|}{ Testing } \\
\hline & & \multicolumn{3}{|l|}{ MEEI } & \multicolumn{3}{|l|}{ SVD } & \multicolumn{3}{|l|}{ AVPD } \\
\hline & & Proposed & $\begin{array}{l}\text { MFCC } \\
24\end{array}$ & $\begin{array}{l}\text { MDVP } \\
22 \\
\end{array}$ & Proposed & $\begin{array}{l}\text { MFCC } \\
24 \\
\end{array}$ & $\begin{array}{l}\text { MDVP } \\
22 \\
\end{array}$ & Proposed & $\begin{array}{l}\text { MFCC } \\
24\end{array}$ & $\begin{array}{l}\text { MDVP } \\
22 \\
\end{array}$ \\
\hline \multirow[t]{6}{*}{ Training } & $\begin{array}{l}\text { MEEI } \\
(1)\end{array}$ & - & - & - & 67.3 & 57.1 & 48.2 & 63.1 & 53.1 & 45.2 \\
\hline & SVD (2) & 84.7 & 74.3 & 75.3 & - & - & - & 88.3 & 75.3 & 74.5 \\
\hline & $\begin{array}{l}\text { AVPD } \\
\text { (3) }\end{array}$ & 78.4 & 64.3 & 62.1 & 80.5 & 71.2 & 63.7 & - & - & - \\
\hline & $(1)+(2)$ & - & - & - & - & - & - & 87.8 & 76.9 & 72.4 \\
\hline & $(2)+(3)$ & 88.5 & 73.1 & 70.8 & - & - & - & - & - & - \\
\hline & $(1)+(3)$ & - & - & - & 88.1 & 75.6 & 73.1 & - & - & - \\
\hline
\end{tabular}

We also performed experiments with a larger subset of MEEI and compared the performances of some state-of-the-art methods. The subset contains samples from 53 normal speakers and 173 pathological speakers (70 male and 103 female). The names of the 173 files are listed in [27]. The same subset was used in the experiments of [1], [6], [28]. In [28], the authors used MFCC and some complexity measures and noise parameters as features. The accuracies of the methods are shown in Table 4. From the table, we find that the proposed method outperforms the three other methods using MEEI subset. 
Table 4. Accuracies (\%) of the methods using MEEI subset (53 normal and 173 pathological).

\begin{tabular}{|l|l|l|l|}
\hline Proposed & Method in [1] & Method in [6] & Method in [28] \\
\hline 99.38 & 94.07 & 94.1 & 98.23 \\
\hline
\end{tabular}

To investigate the influence of the time duration in pathology detection, we performed an experiment, where all the samples the MEEI subset (53 normal and 173 pathological) were set to have the same length. In this way, all the samples had length of $0.9 \mathrm{~s}$; any sample having more than $0.9 \mathrm{~s}$ of length were reduced to first $0.9 \mathrm{~s}$ by truncating the rest. In the experiment, we did not notice a significant change in the detection accuracy; we found the detection accuracy of $99.30 \%$, which is very close to $99.38 \%$ obtained by keeping the samples in their original lengths (see Table 4).

We performed another set of experiments to investigate the influence of using glottal excitation source in the proposed method. In this case, we omitted the block 'inverse filtering' in Fig. 1; so, we applied the 'first-order derivative' directly on the input voice. All other parameters remained the same. Table 5 shows the accuracies of the method in the three databases. From the results, we find that the proposed method applied on the glottal excitation source rather than applied directly on the input voice has a positive effect in the detection of vocal fold pathology.

Table 5. Accuracy $(\%) \pm$ standard deviation of the proposed method with and without using 'inverse filtering' in the three databases.

\begin{tabular}{|c|c|c|c|}
\hline Proposed Method & MEEI & SVD & AVPD \\
\hline $\begin{array}{c}\text { With inverse } \\
\text { filtering }\end{array}$ & $99.4 \pm 0.02$ & $93.2 \pm 0.01$ & $91.5 \pm 0.09$ \\
\hline $\begin{array}{c}\text { Without inverse } \\
\text { filtering }\end{array}$ & $99.1 \pm 0.02$ & $92.4 \pm 0.02$ & $90.2 \pm 0.10$ \\
\hline
\end{tabular}




\subsubsection{Pathology classification}

Fig. 5 shows the classification accuracies of the methods when the same database was used for training and testing, but the samples were not overlapped between training and testing in an iteration. The proposed method achieves the best accuracy with the SVD; cyst has the highest accuracy, followed by paralysis and polyp. In all three databases and all the three pathological cases, the proposed method performs far better than the other two methods (significant at 95\% confidence interval). The IDP-based method gives $98.2 \%, 95.3 \%$, and $94.2 \%$ accuracies for cyst, paralysis, and polyp, respectively, when the AVPD was used. Table 6 shows a confusion matrix of the proposed method. From the confusion matrix, we find that cyst and polyp were confused between each other by the system, while paralysis were equally confused with cyst and polyp.

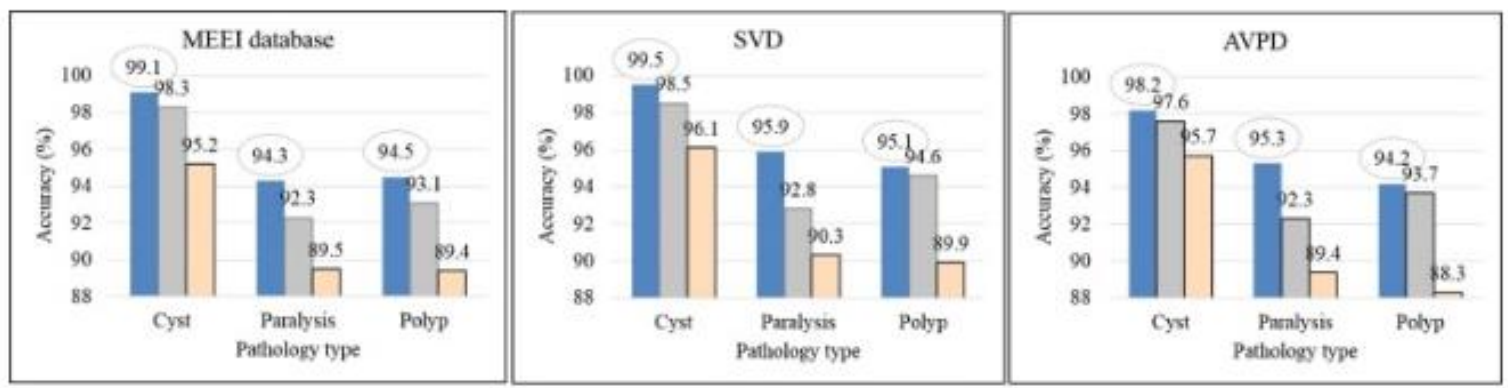

Proposed $\square$ MFCC 24 $\square$ MDVP 22

Fig. 5. Voice pathology classification accuracy (\%) of the methods using the same database training and testing (samples are not overlapped between training and testing in the same iteration). 
Table 6. Confusion matrix of the proposed method for voice pathology classification.

\begin{tabular}{|l|l|l|l|l|l|l|l|l|l|}
\hline & \multicolumn{2}{|l}{ MEEI } & SVD & AVPD \\
\hline Output $\rightarrow$ & Cyst & Paralysis & Polyp & Cyst & Paralysis & Polyp & Cyst & Paralysis & Polyp \\
\hline Input $\downarrow$ & \multicolumn{9}{|l|l|l|l|l|l|l|}{} \\
\hline Cyst & 99.1 & 0 & 0.9 & 99.5 & 0 & 0.5 & 98.2 & 0.4 & 1.4 \\
\hline Paralysis & 2.6 & 94.3 & 3.1 & 3.4 & 95.9 & 0.7 & 1.4 & 95.3 & 3.3 \\
\hline Polyp & 3.9 & 1.6 & 94.5 & 4.3 & 0.6 & 95.1 & 3.0 & 2.8 & 94.2 \\
\hline
\end{tabular}

Fig. 6 shows the classification accuracies of the methods when the two databases' samples were used for training and the third for training. The objective of this type of experiments is to verify if the methods can really perform pathology classification irrespective to recording place, setup, speakers' native language dependency, etc. The classification accuracy of the proposed method never drops below $80 \%$ (confidence interval $=95 \%$ ) in any of the experiments or in any pathological case. This can definitely prove the robustness of the method considering the fact that the training and the testing datasets are different. The other two methods' accuracies are relatively low, mostly below $80 \%$.

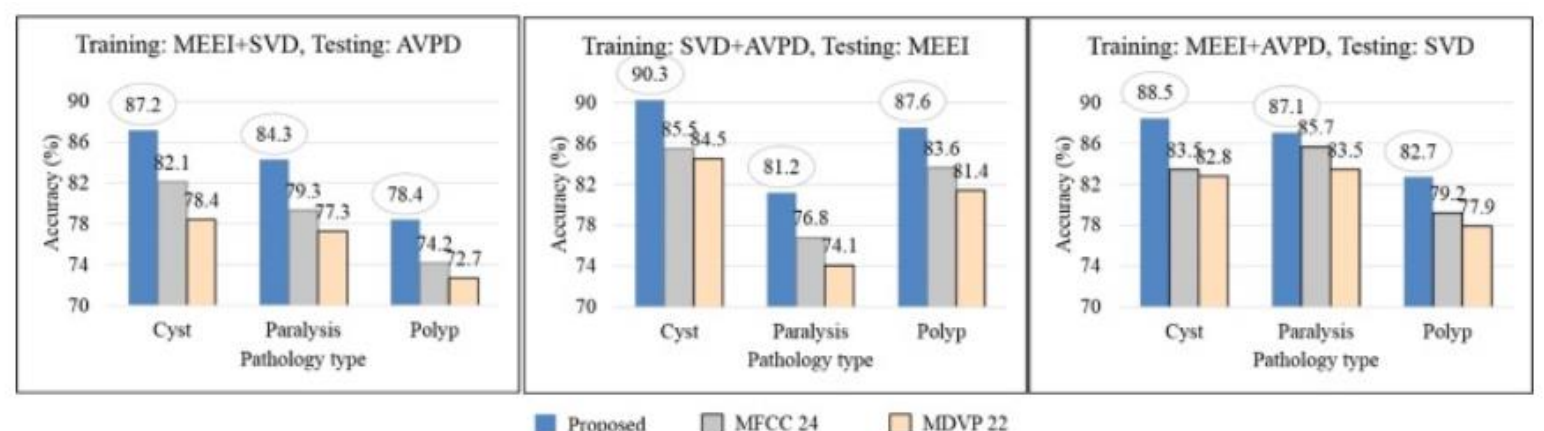

Fig. 6. Voice pathology classification accuracy (\%) of the methods using two databases training and the third database testing. 


\section{Discussion}

An automatic voice pathology detection and classification based on the IDP on the first derivative of the glottal source excitation signal is proposed. First, the glottal source signal is separated from the voice signal by an iterative method, because the glottal source signal can give a clue whether the voice is normal or pathologic. The irregularity of the vocal folds vibration caused by different pathologies produces some weak signals that are associated to the glottal source signal; otherwise, a normal voice produces a fine regular pattern. These weak signals are emphasized by taking the first-order derivative of the glottal source signal. The 2-nd order IDP is calculated on the Mel-spectrogram of the first-order derivative signal. The IDP encodes the relationship along temporal, rising temporal-frequency, frequency, and falling temporal-frequency patterns.

Qiu et al. discovered that some neurons in the primary auditory cortex of mammals are driven to spectro-temporal patterns [34]. Conventional features like MFCC fails to incorporate spectro-temporal pattern in the same coefficient. Therefore, we need directional derivative that can encode changes along both spectral and temporal domain. A first-order derivative [18], [20] operators can detect the change; however, we need a huge number of features to cover the major directions. The IDP not only uses second-order derivatives, but also encodes all the directions into a compact one. Vocal folds' vibration depends on several factors such as mucus present on the vocal folds tissue, stiffness, tension, muscles in the larynx, closing and opening of the folds, etc. These factors are affected differently for various voice pathologies due to the position and the size of the pathologies. Therefore, the vibration varies from one type of pathology to another. This vibration produces glottal source excitation frequencies, and these frequencies are enhanced by a second-order derivative operator, such as the IDP.

The proposed method was evaluated in three different databases, namely, 
the MEEI database, the SVD, and the AVPD. First, the method was tested on each database separately, and compared with two other methods. In the MEEI database, the proposed method achieved 99.4\% detection accuracy, while in the SVD and the AVPD it gained accuracy of 93.2\% and 91.5\%, respectively. These accuracies are far better than the accuracies obtained by the two other methods. In terms of classification accuracy, the proposed method achieved around $99 \%$ accuracy in case of cyst, and between $94 \%$ and $96 \%$ accuracy in case of paralysis and polyp. In the experiments, we observed that the frequency information between $3 \mathrm{kHz}$ and $5 \mathrm{kHz}$ in case of cyst is different than that in case of paralysis and polyp.

In order to verify the robustness of the proposed method irrespective of the recording environments, speakers' native languages, etc., we performed the inter database experiments, where the training and the testing databases were different. When training with the MEEI database, and testing with another database, the performance is low; this is due to the confusion of the classifier whether the trained models are for normal/pathology or different environments. The performance is increased when two databases were used for training and the third database for testing. In all the cases, the proposed method outperformed the other two methods. We chose only three types of pathologies because these three are common in all the three databases.

There are perceptual characteristics for each voice disorders; some of these characteristics overlap, however, others are more specific for certain disorders. For example, the dysphonia in vocal fold paralysis (unilateral) is characterized mainly by weak and breathy voice character due to incomplete glottis closure during phonation, also diplophonia (double pitch) could be there as the two vocal folds are vibrating in a different rate. Polyp and cyst share almost the same voice characteristics of dysphonia including strained voice character, intermittent voice breaks, and sometimes diplophonia due to mass-difference of the vibrating vocal 
folds. Despite this, a trained clinician can distinguish between cyst and polyp by perceptually measuring the hoarseness and poor voice quality. On the contrary, as an automated method is not as intelligent as a human, there are some confusions between cyst and polyp pathologies in the proposed method (see Table 6); however, this amount of confusion is small (3\%-4\%), because, we believe, the proposed features are highly discriminative.

To visualize the distinguishing capability between normal and pathological samples, and between different types of pathological samples, of the proposed features, we draw some examples in Fig. 4. We showed that different pathologies contribute differently in different frequency bands. Due to the position and the shape of the pathologies, and the presence of mucus, tension, and various stiffness of the vocal folds, the opening and closing of the vocal folds show different behavior during the vibration, and therefore produce different frequencies in the output [36]. These frequencies are captured in different filters. A more in-depth investigation on why a particular pathology affects a specific frequency region is needed, and this will be one of our future works.

To the best of our knowledge, this is the first study of multi-database experiments for detection and classification. In [8], the authors experimented with two databases (cross-database) for pathology detection only; however, to do so, they had to normalize the features, which resulted in performance degradation in a single database experiment. In our proposed method, we use derivatives that eliminate the need for normalization.

\section{Conclusion}

A voice pathology detection and classification method based on IDP of the glottal source was proposed. The method was compared with conventional MFCC and MDVP parameters in three different databases. The proposed method outperformed the other two in all the experiments. In cross-database experiments, 
the accuracies reached up to $88.5 \%$ for detection and $90.3 \%$ for classification. So, the proposed method is to some extent database independent. The proposed work will be extended to encode higher order derivative information to improve the performance in a future study. We will also dig more on the performance comparison based on severity level (AVPD has severity information associated to each sample).

\section{Acknowledgment}

This project was funded by the National Plan for Science, Technology and Innovation (MAARIFAH), King Abdulaziz City for Science and Technology, Kingdom of Saudi Arabia, Award Number (12-MED-2474-02).

\section{References}

[1] J.I. Godino-Llorente, P. Gomes-Vilda, M. Blanco-Velasco, Dimensionality reduction of a pathological voice quality assessment system based on Gaussian mixture models and short-term cepstral parameters, IEEE Trans. Biomed. Eng. 53 (October (10)) (2006) 1943-1953.

[2] M. Shamim Hossain, G. Muhammad, Cloud-assisted speech and face recognition framework for health monitoring, Mob. Netw. Appl. 20 (3) (2015) 391-399, http://dx.doi.org/10.1007/s11036-015-0586-3.

[3] Kay Elemetrics, Multi-Dimensional Voice Program (MDVP) [Computer Program] (2012).

[4] G. Muhammad, M. Melhem, Pathological voice detection and binary classification using MPEG-7 audio features, Biomed. Signal Process. Controls 11 (2014) 1-9.

[5] Z. Ali, I. Elamvazuthi, M. Alsulaiman, G. Muhammad, Detection of voice pathology using fractal dimension in a multiresolution analysis of normal and disordered speech signals, J. Med. Syst. 40 (20) (2016), 10 pages.

[6] M. Markaki, Y. Stylianou, Voice pathology detection and discrimination based on modulation spectral features, IEEE Trans. Speech Audio Process. 19 (7) (2011) 1938-1948.

[7] Kay Elemetrics Corp., Disordered Voice Database, Version 1.03 (CD-ROM), MEEI, Voice and Speech Lab, Boston, MA (October 1994).

[8] M. Markaki, Y. Stylianou, Normalized modulation spectral features for cross-database voice pathology detection, in: Proceedings of InterSpeech, Brighton, U.K., September, 2009.

[9] P. Gomez-Vilda, R. Fernández-Baillo, V. Rodellar-Biarge, V. Nieto Lluis, A. 
Álvarez-Marquina, L. Miguel Mazaira-Fernández, R. Martínez-Olalla, J.I. Godino-Llorente, Glottal Source biometrical signature for voice pathology detection, Speech Commun. 51 (2009) 759-781.

[10] T. Drugman, T. Dubuisson, T. Dutoit, On the mutual information between source and filter contributions for voice pathology detection, Proceedings of InterSpeech (2009).

[11] P. Gomez-Vilda, R. Fernández-Baillo, A. Nieto, F. Díaz, F.J. Fernández-Camacho, V. Rodellar, A. Álvarez, R. Martínez, Evaluation of voice pathology based on the estimation of vocal fold biomechanical parameters, J. Voice 21 (4) (2007) 450476.

[12] Y. Koike, J. Markel, Application of inverse filtering for detecting laryngeal pathology, Ann. Otol. Rhinol. Laryngol. 84 (January-February) (1975) 117124.

[13] M. Rosa, J.C. Pereira, M. Grellet, Adaptive estimation of residue signal for voice pathology diagnosis, IEEE Trans. Biomed. Eng. 47 (January (1)) (2000) 96104.

[14] T. Drugman, B. Bozkurt, T. Dutoit, A comparative study of glottal source estimation techniques, Comput. Speech Lang. 26 (January (1)) (2012).

[15] A. Shobeirinejad, Y. Gao, Gender classification using interlaced derivative patterns, 20th International Conference on Pattern Recognition (ICPR) (2010) 1509-1512.

[16] G. Muhammad, Automatic speech recognition using interlaced derivative pattern for cloud based healthcare system, Clust. Comput. 18 (June (2)) (2015) 795-802.

[17] P. Alku, Glottal wave analysis with pitch synchronous iterative adaptive inverse filtering, Speech Commun. 11 (2-3) (1992) 109-118.

[18] G. Muhammad, T. Mesallam, K. Almalki, M. Farahat, A. Mahmood, M. Alsulaiman, Multi directional regression (MDR) based features for automatic voice disorder detection, J. Voice Elsevier 26 (6) (2012), pp. 817. e19-817. e27.

[19] T. Ahonen, A. Hadid, M. Pietikainen, Face description with local binary patterns: application to face recognition, IEEE Trans. Pattern Anal. Mach. Intell. 28 (December (12)) (2006).

[20] G. Muhammad, M. Masud, A. Alelaiwi, M.A. Rahman, A. Karime, A. Alamri, M. Shamim Hossain, Spectro-temporal directional derivative based automatic speech recognition for a serious game scenario, Multimed. Tools Appl. 74 (14) (2015) 53135327, http://dx.doi.org/10.1007/s11042-014-1973-7.

[21] A. Mahmood, M. Alsulaiman, G. Muhammad, Automatic speaker recognition using multi directional local features (MDLF), Arab. J. Sci. Eng. 39 (5) (2014) 3799-3811.

[22] W.J. Barry, M. P utzer, Saarbrucken Voice Database, Institute of Phonetics,

Univ. of Saarland, 2016 http://www.stimmdatenbank.coli.uni-saarland.de/.

[23] D. Martínez, E. Lleida, A. Ortega, A. Miguel, J. Villalba, Voice pathology detection on the saarbruecken voice database with calibration and fusion of scores using MultiFocal Toolkit, in: Proc. of IberSPEECH 2012, CCIS 328, Springer, Berlin, Heidelberg, 2012, pp. 99-109.

[24] S. Abe, Support Vector Machines for Pattern Classification, Springer-Verlag, Berlin, Heidelberg, New York, 2005.

[25] N. Sáenz-Lechón, J.I. Godino-Llorente, V. Osma-Ruiz, P. Gómez-Vilda, Methodological issues in the development of automatic systems for voice pathology 
detection, Biomed. Signal Process. Control 1 (April (2)) (2006) 120-128.

[26] M.K. Arjmandi, M. Pooyan, M. Mikaili, M. Vali, A. Moqarehzadeh, Identification of voice disorders using long-time features and support vector machine with different feature reduction methods, J. Voice 25 (November (6)) (2011) 275-289.

[27] Parsa, D. Jamieson, Identification of pathological voices using glottal noise measures, J. Speech Lang. Hear. Res. 43 (2) (2000) 469-485.

[28] J. Arias-Londono, J. Godino-Llorente, N. Saen-Lechon, V. Osma-Ruiz, G. Castellanos-Dominguez, Automatic detection of pathological voices using complexity measures, noise parameters and mel-cepstral coefficients, IEEE Trans. Biomed. Eng. 58 (2) (2011) 370-379.

[29] J.H. Hansen, L. Gavidia-Ceballos, J.F. Kaiser, A nonlinear operator-based speech feature analysis method with application to vocal fold pathology assessment, IEEE Trans. Biomed. Eng. 45 (3) (1998) 300-313.

[30] J.B. Alonso, J. De Leon, I. Alonso, M.A. Ferrer, Automatic detection of pathologies in the voice by HOS based parameters, EURASIP J. Appl. Signal Process. 4 (2001) 275-284.

[31] M.A. Little, P.E. McSharry, S.J. Roberts, D.A. Costello, I.M. Moroz, Exploiting nonlinear recurrence and fractal scaling properties for voice disorder detection, Biomed. Eng. Online 6 (1) (2007) 23, http://dx.doi.org/10.1186/ 1475-925X-6-23.

[32] P. Henríquez, J.B. Alonso, M.A. Ferrer, C.M. Travieso, J.I. Godino-Llorente, F. Díaz-de-María, Characterization of healthy and pathological voice through measures based on nonlinear dynamics, IEEE Trans. Audio Speech Lang. Process. 17 (6) (2009) 1186-1195.

[33] A. Akbaria, M. Khalil, Arjmandi, Employing linear prediction residual signal of wavelet sub-bands in automatic detection of laryngeal pathology, Biomed. Signal Process. Control 18 (2015) 293-302.

[34] A. Qiu, C. Schreiner, M. Escabi, Gabor analysis of auditory midbrain receptive fields: spectro-temporal and binaural composition, J. Neurophysiol. 90 (2003) 456-476.

[35] G. Muhammad, M. Alsulaiman, A. Mahmood, Z. Ali, Automatic voice disorder classification using vowel formants, in: IEEE International Conference on Multimedia and Expo (ICME)—Workshop MUST-EH 2011, Barcelona, July, 2011.

[36] A. Al-nasheri, G. Muhammad, M. Alsulaiman, Z. Ali, Investigation of voice pathology detection and classification on different frequency regions using correlation functions, J. Voice (2016), http://dx.doi.org/10.1016/j.jvoice.2016. 01.014 . 